\title{
Prevotella heparinolytica
}

National Cancer Institute

\section{Source}

National Cancer Institute. Prevotella heparinolytica. NCI Thesaurus. Code C86669.

A species of obligately anaerobic, Gram-negative, rod shaped bacteria assigned to the phylum Bacteroidetes. This species is nonmotile, non-spore forming, nonpigmented, xylose, arabinose, cellobiose, salicin, sucrose, lactose, indole, esculin hydrolysis, alphafucosidase, beta-xylosidase, beta-glucosidase, and glycine aminopeptidase positive. P. heparinolytica has been isolated from the oral cavity and is associated with oral infections. 